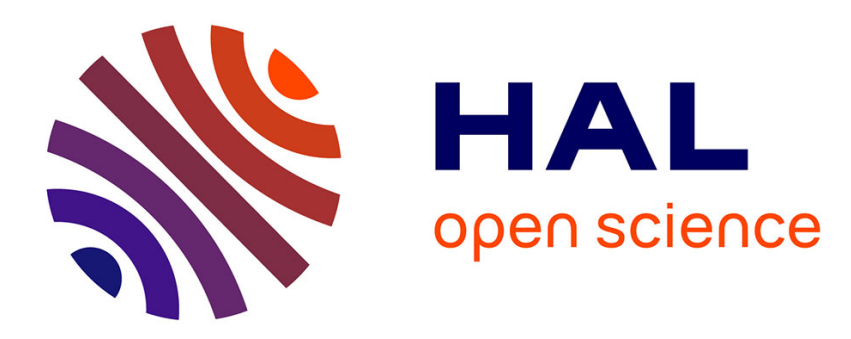

\title{
Mesure de vitesses acoustiques par anémométrie laser dans un tube de Rijke
}

\author{
T. Loizeau, Y. Gervais
}

\section{To cite this version:}

T. Loizeau, Y. Gervais. Mesure de vitesses acoustiques par anémométrie laser dans un tube de Rijke. Journal de Physique IV Proceedings, 1994, 04 (C5), pp.C5-973-C5-976. 10.1051/jp4:19945213 . jpa00252898

\section{HAL Id: jpa-00252898 https://hal.science/jpa-00252898}

Submitted on 1 Jan 1994

HAL is a multi-disciplinary open access archive for the deposit and dissemination of scientific research documents, whether they are published or not. The documents may come from teaching and research institutions in France or abroad, or from public or private research centers.
L'archive ouverte pluridisciplinaire HAL, est destinée au dépôt et à la diffusion de documents scientifiques de niveau recherche, publiés ou non, émanant des établissements d'enseignement et de recherche français ou étrangers, des laboratoires publics ou privés. 


\title{
Mesure de vitesses acoustiques par anémométrie laser dans un tube de Rijke
}

\author{
T. LOIZEAU et Y. GERVAIS
}

Laboratoire d'Etudes Aérodynamiques, 40 Avenue du Recteur Pineau, 86022 Poitiers cedex, France

\begin{abstract}
We present some results of experimental investigations on thermoacoustic phenomemon, and more particularly on the Rijke's phenomenon. The goal is to give an experimental description of the instantaneous heat release between a hot grid and an acoustic wave leading to self excitation into a two open ended duct. As the unsteady thermal flux is directly related to the instantaneous velocity, measurements of the acoustic and mean velocities are made using the Laser Doppler Velocimetry method. This allows an evaluation of the unsteady heat released, and so of the amplitude of the thermoacoustic waves from theoretical models, which can then be compared to experimental results.
\end{abstract}

\section{INTRODUCTION}

Les phénomènes thermoacoustiques résultent de l'interaction entre une onde acoustique et un transfert thermique. Ils sont classés selon leur mode d'évolution en deux catégories distinctes: phénomène de Sondhauss et phénomène de Rijke. Nos travaux concernent principalement l'étude du phénomène de Rijke, qui apparaît dans les cavités résonnantes contenant une source thermique et parcourues par un écoulement. Lorsque la puissance thermique est suffisamment élevée, des oscillations acoustiques de fortes amplitudes s'établissent et s'auto-entretiennent sous l'effet du couplage thermoacoustique.

L'objet de ce travail est d'examiner expérimentalement les principales caractéristiques quantitatives de ce couplage qui dépend de l'échange de chaleur instationnaire entre la source thermique et l'onde acoustique. Les quantités de chaleur échangées, et donc le niveau acoustique engendré, sont fortement conditionnés par la vitesse de l'écoulement et l'amplitude des oscillations de vitesse acoustique au voisinage de l'élément chauffant [1]. Ceci nous a conduit à concevoir un banc expérimental de mesure de vitesses moyenne et acoustique à l'intérieur d'une cavité résonnante dans laquelle se déclenchent spontanément et s'auto-entretiennent des oscillations thermoacoustiques. Les mesures effectuées permettent de quantifier les principales caractéristiques de ce couplage afin d'évaluer les quantités de chaleur échangées et donc la puissance thermoacoustique développée [2].

\section{BANC EXPERIMENTAL}

Nous avons développé et mis au point un banc expérimental permettant l'auto-déclenchement et l'auto-entretient du phénomène thermoacoustique de Rijke, en positionnant une grille chauffante à l'intérieur d'un tube vertical en verre ouvert à ses deux extrémités. La grille chauffante est située au quart inférieur du tube en verre. On suit l'évolution du phénomène (déclenchement, auto-entretient...) par la mesure des principales grandeurs qui le caractérisent, c'est à dire la pression acoustique à l'intérieur du tube, les oscillations de vitesse instantanée, la puissance électrique d'alimentation où la température de la grille chauffante. La pression est mesuré à l'intérieur du tube par un microphone B\&K placé en paroi, au milieu du tube et connecté soit à un analyseur B\&K, soit à un analyseur 'temps réel' type OROS. La température de la grille chauffante est mesurée par un thermocouple soudé en un point de la grille et connecté à un boîtier électronique de mesure. 
Les mesures de vitesse sont rélisées par une chaîne Anémométrique Laser à effet Doppler connectée à un micro-ordinateur qui réalise l'acquisition et le traitement des informations numériques délivrées par le compteur de périodes. Cette technique de mesure a été choisie afin de bénéficier de ses possibilités nonintrusives, d'écarter les problèmes thermiques rencontrés dans le phénomène thermoacoustique et de pouvoir pallier à d'éventuelles inversions de la vitesse instantanée. Pour réaliser ces mesures, le tube de Rijke est placé dans un volume de confinage à l'intérieur duquel l'air est ensemencé par un brouillard de fines particules d'huile, de telle sorte que le développement du phénomène ne soit pas perturbé.

La méthode de mesure de l'amplitude des oscillations de vitesse a été validée à partir de la mesure de la vitesse acoustique instantanée dans un tube à ondes stationnaires excité par un haut-parleur.

\section{TRAITEMENT DES DONNEES L.D.A.}

Pour étudier l'évolution des vitesses moyenne et acoustique d'un fluide, nous disposons principalement des deux représentations complémentaires suivantes: représentation temporelle et représentation fréquentielle. Les algorithmes de traitement informatique classiques réalisant le passage d'un espace à l'autre (espace temporel, espace fréquentiel) à l'aide de la transformée de Fourier où de son inverse ne sont pas applicables aux signaux à pas non constants délivrés par la chaîne de mesure Laser [3]. En effet, le signal fourni par le compteur de périodes est échantillonné de manière totalement aléatoire puisqu'il dépend directement de la présence de particules de fluide dans le volume de mesure [4]. Ceci nous impose de développer des modes de traitement appropriés.

Dans l'espace temporel, la représentation du signal est directement accessible à partir des données provenant du compteur de périodes qui fournit, pour chaque point acquis, l'amplitude et le temps qui le sépare du point précédent. La difficulté apparaît lorsqu'on souhaite réaliser la représentation fréquentielle de ce signal qui est échantillonné par principe de manière totalement aléatoire. Ainsi, pour réaliser cette représentation à partir du signal à pas temporels non constants, nous avons développé un calcul spectral particulier basé sur la relation générale de Fourier, tout en respectant le théorème de Shannon.

La théorie de Fourier montre qu'un signal quelconque $g(t)$ peut-être décomposé en une somme de signaux sinusoïdaux dont l'amplitude en fonction de la fréquence est donnée par la relation suivante :

$$
G(f)=\int_{-\infty}^{+\infty} g(t) \cdot e^{-j .2 . x . f . t} \cdot d t \quad G(f) \text { est la transformée de Fourier de } g(t)
$$

L'expression du signal échantillonné s'écrit : $g^{\mathrm{e}}(\mathrm{t})=\mathrm{g}(\mathrm{t})$.Pgn(t) où la fonction Peigne de Dirac 'Pgn(t)' qui échantillonne la fonction continue $g(t)$ est considérée comme une suite aléatoire de distribution de Dirac.

On définit l'expression de la transformée de Fourier de la fonction échantillonnée à pas non constants ' $g$ e $(t)$ ' par :

$$
G^{e}(f)=\int_{-\infty}^{+\infty} g^{e}(t) \cdot e^{-j \cdot 2 \cdot \pi \cdot f \cdot t} \cdot d t
$$

Compte tenu de la valeur finie du temps d'acquisition des séquences étudiées, cette expression devient l'estimation suivante :

$$
\hat{G}^{e}(f)=\frac{1}{T_{a c q}} \int_{i_{0}}^{t_{0}+T_{m q}} g^{e}(t) \cdot e^{-j \cdot 2 . \pi . f . t} \cdot d t \quad \text { avec } \quad T_{a c q}=\sum_{n=0}^{N} \Delta t_{n}
$$

qui en valeurs discrètes s'exprime :

$$
\hat{G}^{e}(f)=\frac{1}{N+1} \sum_{n=0}^{N}\left(g^{e}\left(t_{n}\right) \cdot e^{-j \cdot 2 \cdot x \cdot f \cdot t_{n}}\right)
$$

Pour calculer l'amplitude des oscillations de vitesse acoustiques, on applique cette relation aux 
données provenant du compteur de périodes. Pour affiner la précision des résultats, on utilise des fenêtres de pondération et on retranche la composante continue à chaque séquence étudiée.

\section{RESULTATS EXPERIMENTAUX}

\subsection{Validation de la méthode de mesure}

Nous avons tout d'abord validé la méthode de mesure et de traitement en comparant les résultats obtenus lorsque le tube est excité à la résonance par une source acoustique extérieure, aux valeurs calculées à partir de la mesure de la pression acoustique au centre du tube.

La première représentation graphique (Figure:1), montre un exemple type de l'évolution de la vitesse instantanée des particules de fluide qui traversent le volume de mesure. Ces informations délivrées par le compteur de périodes montrent la bonne qualité de la définition de l'écoulement par ce procédé de mesure et permettent d'estimer les valeurs instantanées ordres de grandeur de la vitesse moyenne et des caractéristiques principales de la fluctuation acoustique prépondérante. La figure 2 présente la densité spectrale du signal précédent que nous avons obtenue par le calcul de la transformée de Fourier à pas non constants qui nous permet d'obtenir la valeur de l'amplitude et la fréquence de la fluctuation acoustique principale.

Ces informations nous permettent d'établir l'évolution de l'amplitude de la vitesse acoustique stationnaire le long du tube dans lequel se développe le phénomène de résonance acoustique. Ces résultats, présentés sur la figure 3, montrent la bonne qualité de la mesure dont les valeurs restent très proches de celles calculées avec une erreur relative inférieure à cinq pour-cent.

\subsection{Résultats relatifs au phénomène thermoacoustique}

Les figures 4 et 5 presentent respectivement une évolution temporelle et une répartition fréquentielle de la vitesse acoustique au niveau de la grille chauffante lorsque le phénomène est déclenché. Le dernier graphique (Figure:6) montre l'évolution de l'amplitude de l'onde acoustique stationnaire à l'intérieur du tube de Rijke et à ses extrémités, en présence là encore du phénomène thermoacoustique. On note sur cette représentation de l'évolution de l'amplitude de l'onde acoustique pour trois puissances différentes d'alimentation de la grille chauffante que le niveau croît avec la puissance. On constate que la répartition de la vitesse acoustique n'est plus symétrique par rapport au milieu du tube et que l'amplitude de l'onde aux deux extrémités est nettement différente. Ceci dépend principalement du saut de température qui existe au niveau de la grille chauffante, entre les parties amont et aval du tube. Cette représentation indique également que l'amplitude décroît légèrement avant les extrémités du tube, pour chuter complètement à l'extérieur de celui-ci.

\section{CONCLUSION}

L'ensemble des résultats obtenus montre que l'anémométrie Laser à effet Doppler est bien adaptée à la mesure de fluctuations de vitesse acoustiques. Elle permet d'accéder à des valeurs très faibles avec un taux de résolution très satisfaisant.

L'utilisation de cette technique pour l'étude des phénomènes thermoacoustiques a fournit de nombreuses informations sur la vitesse instantanée au niveau de la grille, permettant ainsi de déterminer théoriquement les quantités de chaleur échangées et donc la puissance thermoacoustique issue du couplage. Ces mesures, associées à des mesures de pression aux extrémités du tube, permettent de déterminer l'impédance de rayonnement et donner ainsi des informations sur l'énergie acoustique rayonnée vers l'extérieur du tube. Ces résultats permettent par la suite d'établir des bilans d'énergie acoustique pour l'ensemble du tube, et d'évaluer l'amplitude limite des oscillations thermoacoustiques.

\section{REFERENCES}

[1] KWON Y.P. and LEE B.H.,"Stability of the Rijke thermoacoustic oscillation"; J.A.S.A. (1985)

[2] HECKL M.A., "Non-linear acoustic effects in the Rijke tube"; ACUSTICA, Vol.72, (1990)

[3] DANTEC, "Anémométrie Doppler Laser"; Cours L.D.A.3,(1986)

[4] MAX J., "Méthodes et techniques de traitement du signal et applications aux mesures physiques" 


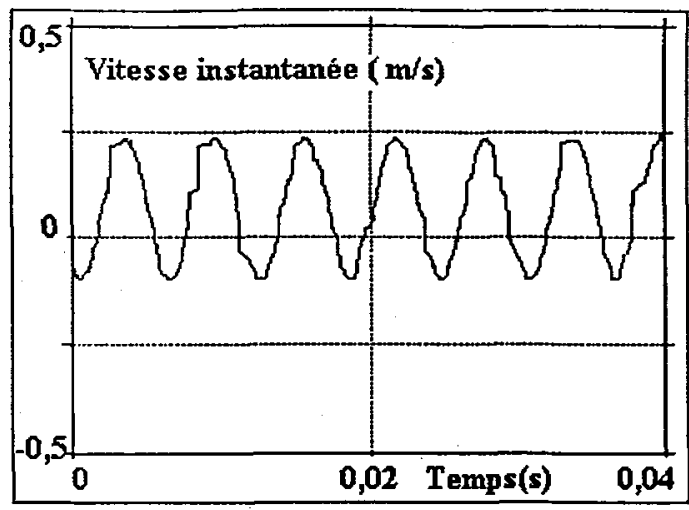

Figure 1: Vitesse instantanee $(\mathrm{m} / \mathrm{s})$

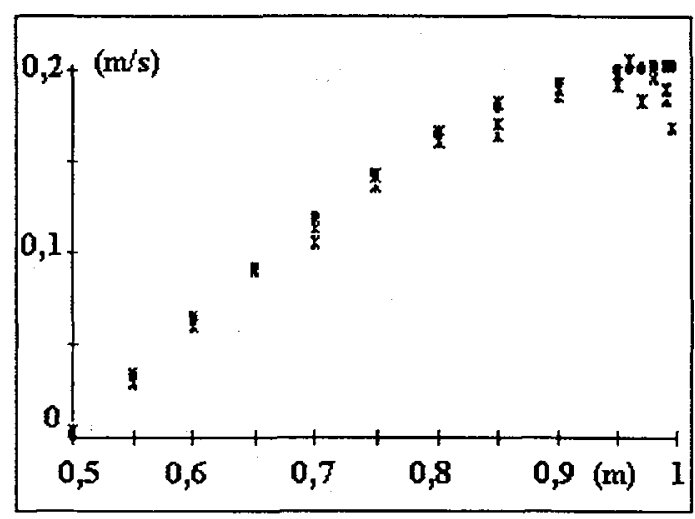

Figure 3: Vitesse acoustique le long du tube - : vitesse théorique; $*$ : vitesse mesurée

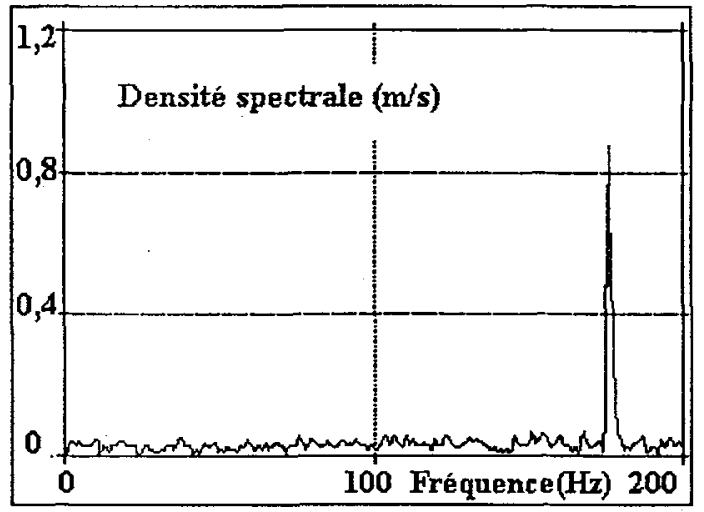

Figure 5: Densité spectrale $(\mathrm{m} / \mathrm{s})$

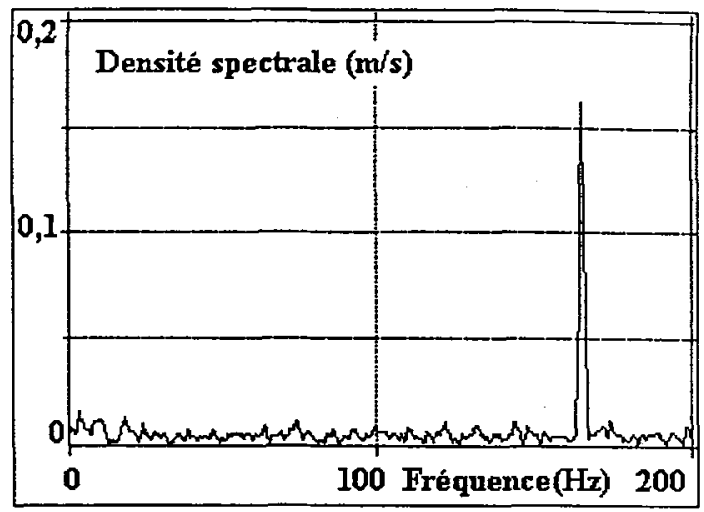

Figure 2: Densité spectrale $(\mathrm{m} / \mathrm{s})$

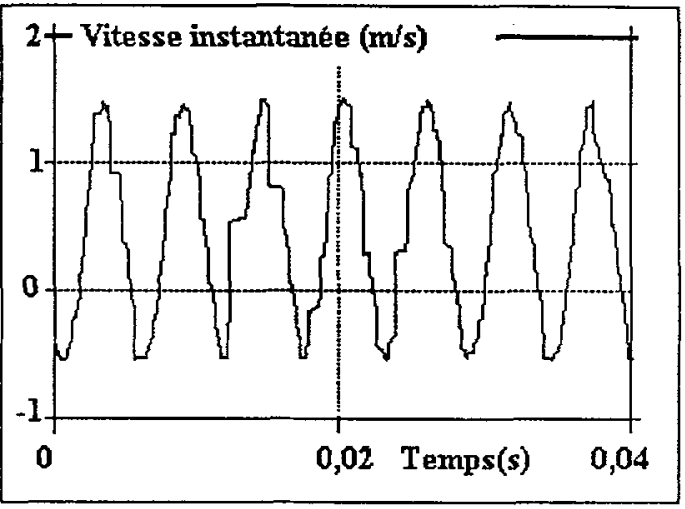

Figure 4: vitesse instantanée $(\mathrm{m} / \mathrm{s})$

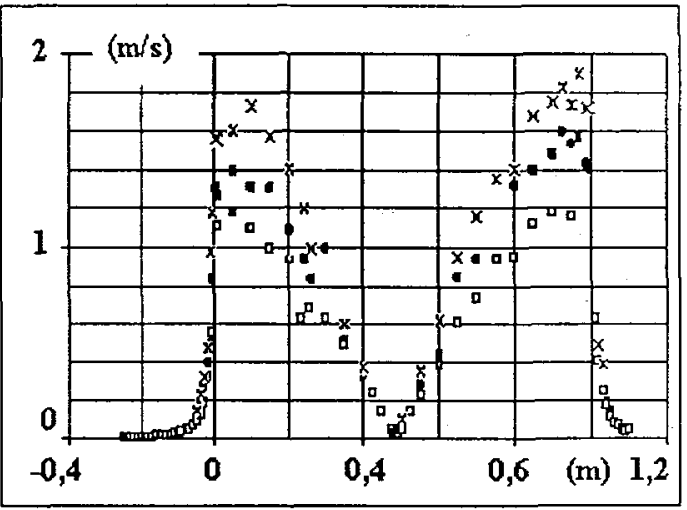

Figure 6: Vitesse acoustique le long du tube $\square: 95$ Watts; $: 135$ Watts; $\times$ : 180 Watts 